\title{
Constitutive Relation Research of Q235 Steel Based on Support Vector Machine
}

\author{
Gening $X u^{1, a}$, Ying Liu ${ }^{2, b}$, Ning $X u^{3, c}$ \\ School of Machinery Engineering, Taiyuan University of Science and Technology, \\ Taiyuan 030024, China \\ a xugening@sina.com, ${ }^{\mathrm{b}} 1243607831 @ q q . c o m$
}

\begin{abstract}
Keywords: Constitutive relation, Support vector machine, Mapping model, Neural network, Nonlinear relationship

Abstract. Aiming at the problem of high cost of mechanics performance testing and uncontrollable of the experimental accuracy, a new modeling method which called "mapping model" based on Support Vector Machine (SVM) is proposed. The SVM regression nonlinear prediction model was established based on the small sample of experimental data by taking the different temperatures of Q235 tensile test as input parameters, the elastic modulus (ultimate strength, yield strength) as output parameters. By MATLAB programming, stress-strain properties of Q235 steel were calculated and analyzed for different temperatures referring to British standard, the constitutive relation which is convenient for engineering use are obtained by fitting the small sample of experimental data and forecast data. The research shows that constitutive relation equations based on experimental data and forecast data could show a complex nonlinear relationship between mechanical properties and temperature. At the same time, according to the comparison of mapping results which is predicted by SVM and neural network, it comes the conclusion that prediction model via SVM have the advantage of less relative error, fewer calculation, high goodness of fit.
\end{abstract}

\section{Introduction}

With the rapid development of China's economy, steel production and quality has been greatly improved. Crude steel output in China accounted for almost half t global output. Because of its good mechanical performance, complete specifications, low environmental pollution, high reliability, low price is cheap and satisfying the requirement of structure, Q235 is widely used ${ }^{[1]}$, which is the common material of main component of mechanical equipment. Due to the growing complexity of crane working environment, including at low temperatures and under high temperature conditions, the steel's mechanical properties such as yield strength, ultimate strength and elastic modulus are changing with temperature change.

Traditional mechanical properties test is measured by material testing equipment which is in accordance with the test methods and procedures prescribed, its cost is relatively high, and the experimental accuracy is easily affected. In this paper, it establishes the mapping modeling method via Support Vector Machine, which is designed for small sample data and based on the statistical learning theory. By introducing the kernel function, we train the input and output sample data to form a nonlinear relationship between the input and the output .so as to establish a scientific and reasonable, high precision, less computing time, low distortion degree model of "mapping model"[2].

In this paper, taking constitutive relation of Q235 steel as the object, referring to British standard, stress - strain properties of Q235 steel were calculated and analyzed for different deformation temperatures, in order to obtain the constitutive relation which is convenient for engineering use.

\section{Test condition ${ }^{[3-5]}$}

\subsection{Test purpose}

(1)To obtain the stress-strain relationship of Q235 steel, yield strength, ultimate strength, elastic modulus and other mechanical properties under different temperatures.

(2)The law of mechanical properties of Q235 steel with temperature changes was fit with mathematical method, and compare with the support vector machine. 


\subsection{Test conditions and test procedure description}

Q235 performance test at different temperatures was carried out on the SUNS of the mechanical test center of Taiyuan University of Science and Technology. The maximum loading capacity of the test machine is $11 \mathrm{kN}$, and the maximum heating temperature is $1200^{\circ} \mathrm{C}$, and can be completed the one-way loading under constant temperature, and the stress-strain curve of the test piece can be automatically recorded. The experiment was carried out strictly in accordance with the GB/T228.1-2010 "Tensile Test of Metal Materials" and the GB/T4338-2006 "high temperature tensile testing method for metal materials". Try to specify the shape of the test machine, dimensions, and size is shown in figure 1.

The longitudinal tensile test of Q235 steel at different temperatures was carried out. Test

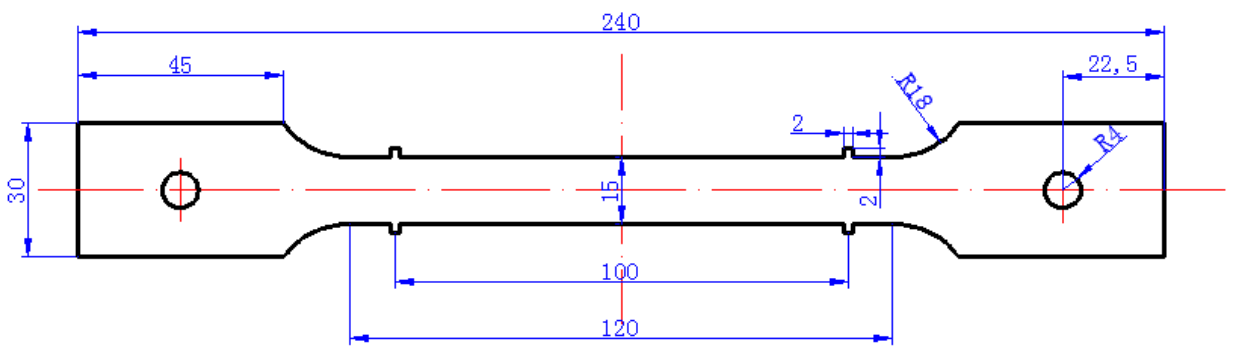

Figure 1 Dimensions of tensile test specimen (unit:mm)

temperature consists of $-60^{\circ} \mathrm{C},-40^{\circ} \mathrm{C},-20^{\circ} \mathrm{C}, 0^{\circ} \mathrm{C}, 20^{\circ} \mathrm{C}, 100^{\circ} \mathrm{C}, 200^{\circ} \mathrm{C}, 300^{\circ} \mathrm{C}, 400^{\circ} \mathrm{C}, 500^{\circ} \mathrm{Cand} 600^{\circ} \mathrm{C}$, a total of 11 points. 3 specimens were measured under normal temperature, and 2 specimens were measured at other temperatures. The main data of the test include stress-strain relationship, yield strength, ultimate strength and elastic modulus. Because of the accuracy of testing apparatus, the measurement data of the elastic modulus are bigger. The test specimens are 23.

Through the analysis and observation of experimental data, when the temperature is not higher than $200^{\circ} \mathrm{C}$, Q235 elastic modulus and yield strength remains unchanged, and the phenomenon of blue brittle is at $300^{\circ} \mathrm{C}$, the stress-strain relationship of steel has no obvious yield platform as shown in figure 2 .

\section{Mapping model of Q235 steel}

\subsection{Theory of Support Vector Machine ${ }^{[6-9]}$}

SVM (Support Vector Machine) theory was proposed by Vapnik in 1963 to solve the problem of pattern recognition. This method selects a set of features from the training set subset, which is equivalent to the entire set of segmentation. This set of feature subsets called support vectors SV (Support Vector). SVM has the obvious advantages of generalization ability, no local minimum value and sparse expression.

SVM is by using nonlinear transformations of the

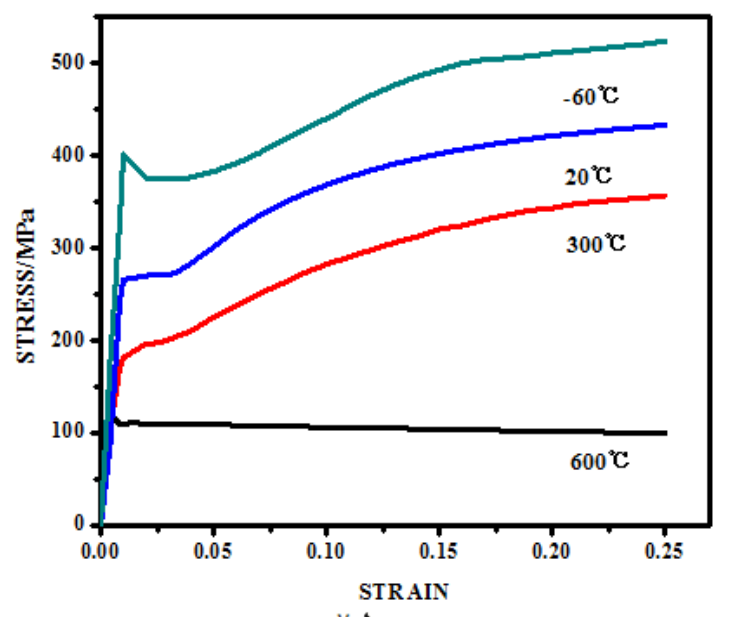
inner product function which transformed input space into a high-dimensional space, so as to find the generalized optimal classification plane. SVM classification function is similar to a neural network, the output is a linear combination of intermediate nodes, each node corresponds to a support vector, as shown in Figure 3. $x^{1} \sim x^{d}$ is input data for training, which represents the temperature of Q235 constitutive relations, $y$ is output data, which represent the elastic modulus, ultimate strength, yield strength of Q235 carbon structural steel. $K\left(x_{i}, x\right)$ is kernel function of 
support vector machine, which play an important role on the product operation $\Phi\left(x_{i}\right) \cdot \Phi\left(x_{j}\right)$ of 2 points in high-dimensional Hilbert space.

Classification method of support vector machine is simple and important, the data in the training data set is linearly inseparable, in order to solve this problem, kernel function is applied, whose function is to transform the sample into a high-dimensional space, and turn it into a linear regression problem. In practical applications, the most commonly used kernel functions are polynomial kernel functions, Gaussian Radial Basis Functions (RBF), Sigmiod kernel functions, exponential kernel, B-spline kernel, and so on.

The generalization performance of the SVM regression problem is determined by a set of good parameters, such as the regularization parameter $\mathrm{C}$ and insensitive parameter $\varepsilon$. The regularization parameter $\mathrm{C}$ can be used to make a tradeoff between the complexity and the training error, so that the model has good generalization ability. Searching for the appropriate $\mathrm{C}$ in each data space can make the SVM generalization performance best. Insensitive parameters $\varepsilon$ control the width of the $\varepsilon$-insensitive band, which affects the number of support vectors. Therefore, in the standard SVM, the parameters $\varepsilon$ and $\mathrm{C}$ control the complexity of the model in different means.

\subsection{Implementation steps of $Q 235$ constitutive relation mapping modeling}

MATLAB software comes with the optimization toolbox and MATLAB program is developed based on the platform for user visual and convenient for training and forecasting data. Steps for:

(1)Select the appropriate training sample. In order to make the results as accurate as possible, the five experimental datas were selected from the experimental data on both sides and arbitrary three sets of data which temperature was as the input, the yield strength(ultimate strength, elastic modulus) was as the output. The remaining temperature datas were as forecast.

(2)Solve the optimization problem and establish SVM.m files, according to the MATLAB;

(3)Select the appropriate sensitivity coefficient $\varepsilon$, penalty factor $\mathrm{C}$ and kernel function, $\varepsilon=0.0001, \mathrm{C}=49$,after repeating comparison of the kernel function result, the final type of kernel function is exponential kernel, as formula (1), then (1) set the training samples in programs (1) into programs $(2)$ :

$$
K\left(x, x^{\prime}\right)=\exp \left(-\frac{\left\|x-x^{\prime}\right\|}{2 \sigma^{2}}\right)
$$

(4)To avoid the calculation of saturation phenomenon, it is necessary to "normalized" input and output variables ,according formula (2) so that it falls on the $[0,1]$.which which temperature is as the input vector of SVM model, the elastic modulus E, yield strength $\sigma_{\mathrm{s}}$, ultimate strength $\sigma_{\mathrm{b}}$ are as the output vector.

$$
X^{\prime}=2\left(\frac{X-X_{\min }}{X_{\max }-X_{\min }}\right)-1
$$

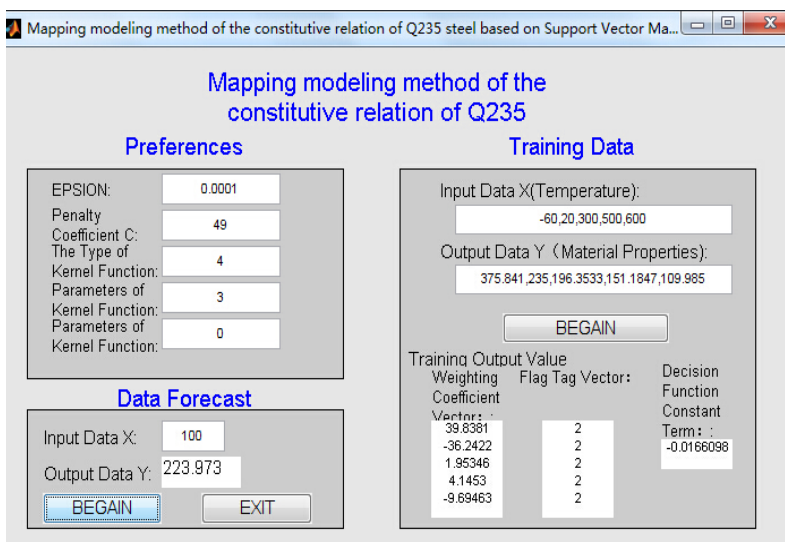

(5)Design graphic user interface, which is constitutive relation of Q235 $\varepsilon$-SVR mapping modeling interface, as shown in figure 4;

Figure 4 Mapping modeling interface of Q235

(6)Test the data that is a part of the training sample test data, judge the quality of training, and adjust $\varepsilon$ and $\mathrm{C}$ to achieve the best training results;

(7)Enter the remaining temperatures, and then obtain the yield strength, ultimate strength and elastic modulus.

\subsection{Analysis of results ${ }^{[10-11]}$}

The sample training sets were selected from five different temperatures of $-60^{\circ} \mathrm{C}, 20^{\circ} \mathrm{C}, 300^{\circ} \mathrm{C}$, $500^{\circ} \mathrm{Cand} 600^{\circ} \mathrm{C}$, and the mapping model was performed. Adjust $\varepsilon$ and $\mathrm{C}$ to achieve the best training results, predicts the remaining temperatures, and then obtains the corresponding yield strength, ultimate strength and elastic modulus. Experimental data, calculation results of the mapping model 
and relative errors are shown in Table1.

Table 1 Comparison between experimental and predicted values

\begin{tabular}{|c|c|c|c|c|c|c|c|c|c|}
\hline \multirow[b]{2}{*}{$\begin{array}{l}\text { Tempera } \\
\text {-ture } /{ }^{\circ} \mathrm{C}\end{array}$} & \multicolumn{3}{|c|}{ Elastic modulus/Pa } & \multicolumn{3}{|c|}{ Yield strength/MPa } & \multicolumn{3}{|c|}{ Ultimate strength/MPa } \\
\hline & test values & $\begin{array}{c}\text { Predicted } \\
\text { values }\end{array}$ & $\begin{array}{c}\text { Error } \\
(\%)\end{array}$ & test values & $\begin{array}{l}\text { Predicted } \\
\text { values }\end{array}$ & $\begin{array}{c}\text { Error } \\
(\%)\end{array}$ & test values & $\begin{array}{l}\text { Predicted } \\
\text { values }\end{array}$ & $\begin{array}{c}\text { Error } \\
(\%)\end{array}$ \\
\hline-40 & $2.06 \mathrm{E}+11$ & $2.06 \mathrm{E}+11$ & -0.003 & 341.22 & 340.627 & -0.174 & 540.846 & 537.725 & -0.577 \\
\hline-20 & $2.06 \mathrm{E}+11$ & $2.06 \mathrm{E}+11$ & -0.003 & 306.51 & 305.423 & -0.355 & 516.217 & 515.344 & -0.169 \\
\hline 0 & $2.06 \mathrm{E}+11$ & $2.06 \mathrm{E}+11$ & -0.003 & 268.15 & 270.22 & 0.772 & 489.314 & 492.964 & 0.747 \\
\hline 100 & $1.99 \mathrm{E}+11$ & $1.94 \mathrm{E}+11$ & -2.636 & 225.01 & 223.973 & -0.461 & 450.894 & 450.138 & -0.168 \\
\hline 200 & $1.80 \mathrm{E}+11$ & $1.78 \mathrm{E}+11$ & -1.07 & 212.23 & 210.163 & -0.974 & 426.841 & 424.598 & -0.53 \\
\hline 400 & $1.44 \mathrm{E}+11$ & $1.44 \mathrm{E}+11$ & 0.065 & 172.84 & 173.769 & 0.537 & 339.84 & 341.21 & 0.403 \\
\hline
\end{tabular}

Steel at high temperatures does not appear obvious yield platform, the British standard shows that the yield stress of the steel is the stress of strain $2 \%$.The results are shown in a tabular, reduction factors at different temperature is relative to the values of yield strength or elastic modulus, and compared with British standards, as shown in table 2.

Table 2 Reduction factors for stress-strain relationships of carbon steel at different temperatures

\begin{tabular}{ccccc}
\hline \multirow{2}{*}{ Temperature $/{ }^{\circ} \mathrm{C}$} & \multicolumn{2}{c}{$\begin{array}{c}\text { Reduction factor for the slope } \\
\text { of the linear elastic range }\end{array}$} & \multicolumn{2}{c}{$\begin{array}{c}\text { Reduction factor for effective } \\
\text { yield strength }\end{array}$} \\
\cline { 2 - 4 } & British standard & Experiment in this paper & British standard & Experiment in this paper \\
\hline-60 & - & 1.000 & - & 1.599 \\
-40 & - & 1.000 & - & 1.452 \\
-20 & - & 1.000 & - & 1.304 \\
0 & - & 1.000 & 1.000 & 1.141 \\
20 & 1.000 & 1.000 & 1.000 & 0.958 \\
100 & 1.000 & 0.966 & 1.000 & 0.903 \\
200 & 0.900 & 0.876 & 1.000 & 0.836 \\
300 & 0.800 & 0.792 & 1.000 & 0.735 \\
400 & 0.700 & 0.700 & 0.780 & 0.643 \\
500 & 0.600 & 0.609 & 0.470 & 0.468 \\
\hline
\end{tabular}

NOTE: For intermediate values of the steel temperature, linear interpolation may be used

\section{Establishment of constitutive equation}

Under limited conditions, accurate experimental data are obtained at specific temperatures, .By forecasting support vector machine, using MATLAB programming, the constitutive equation is obtained by fitting the small sample of experimental data and forecast data.

$$
\left\{\begin{array}{l}
E=2.07 \times 10^{11}-4.67 \times 10^{7} T-1.15 \times 10^{6} T^{2}+3.83 \times 10^{3} T^{3}-4.16 T^{4} \\
\sigma_{s}=275-1.19 T+6.88 \times 10^{-3} T^{2}-1.59 \times 10^{-5} T^{3}+1.16 \times 10^{-8} T^{4} \\
\sigma_{b}=486.19-0.84 T+6.27 \times 10^{-3} T^{2}-2.05 \times 10^{-5} T^{3}+1.78 \times 10^{-8} T^{4}
\end{array},-60{ }^{\circ} \mathrm{C} \leq T \leq 600^{\circ} \mathrm{C}\right.
$$

Where: $E$-Elastic modulus; $\sigma_{s}$-Yield strength; $\sigma_{b}$ —Ultimate strength; $T$-Temperature.

The comparison with fitting curves of constitutive equations of Q235 steel with experimental values is shown in Figure 5. It can be seen that the fitting curve of the equation is in good agreement with experimental data, which can show the complex nonlinear relationship between the temperature and the mechanical properties of Q235 steel. 

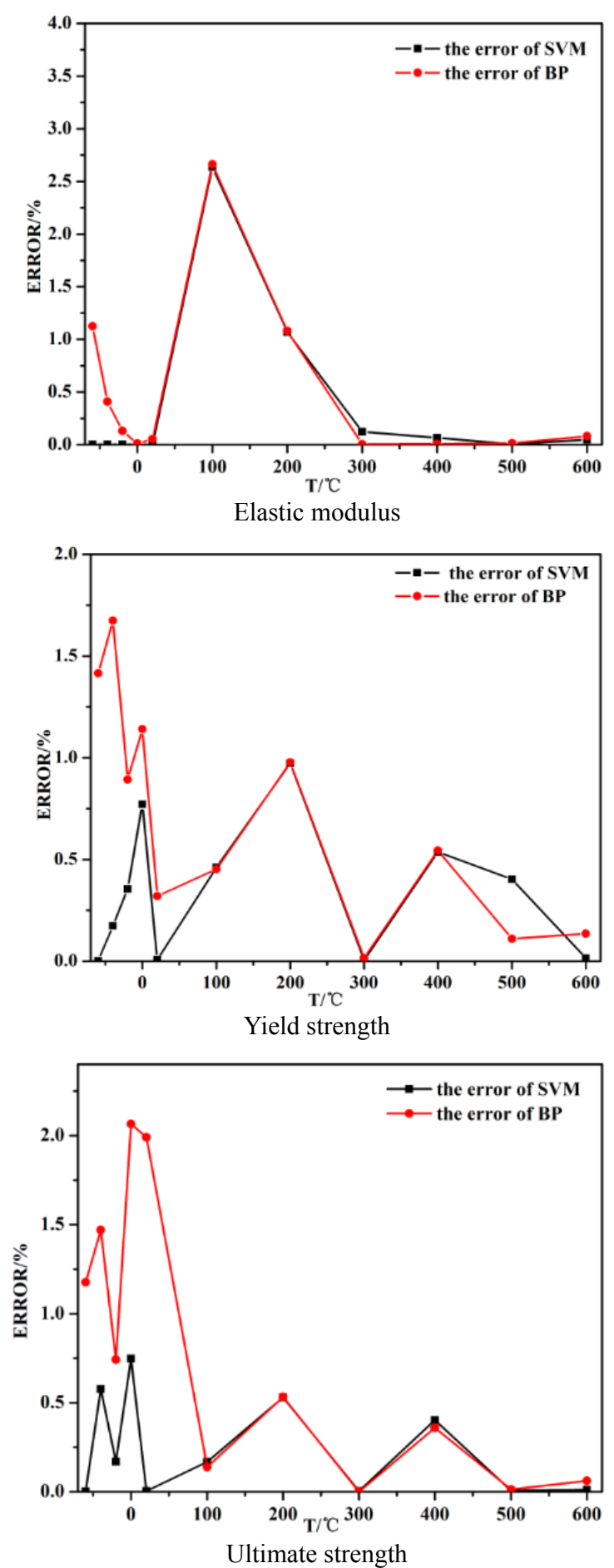

Figure 6 Relative error comparison between SVM and $\mathrm{BP}$ for different deformation temperatures

\section{Comparison of mapping modeling methods between support vector machine and neural network}
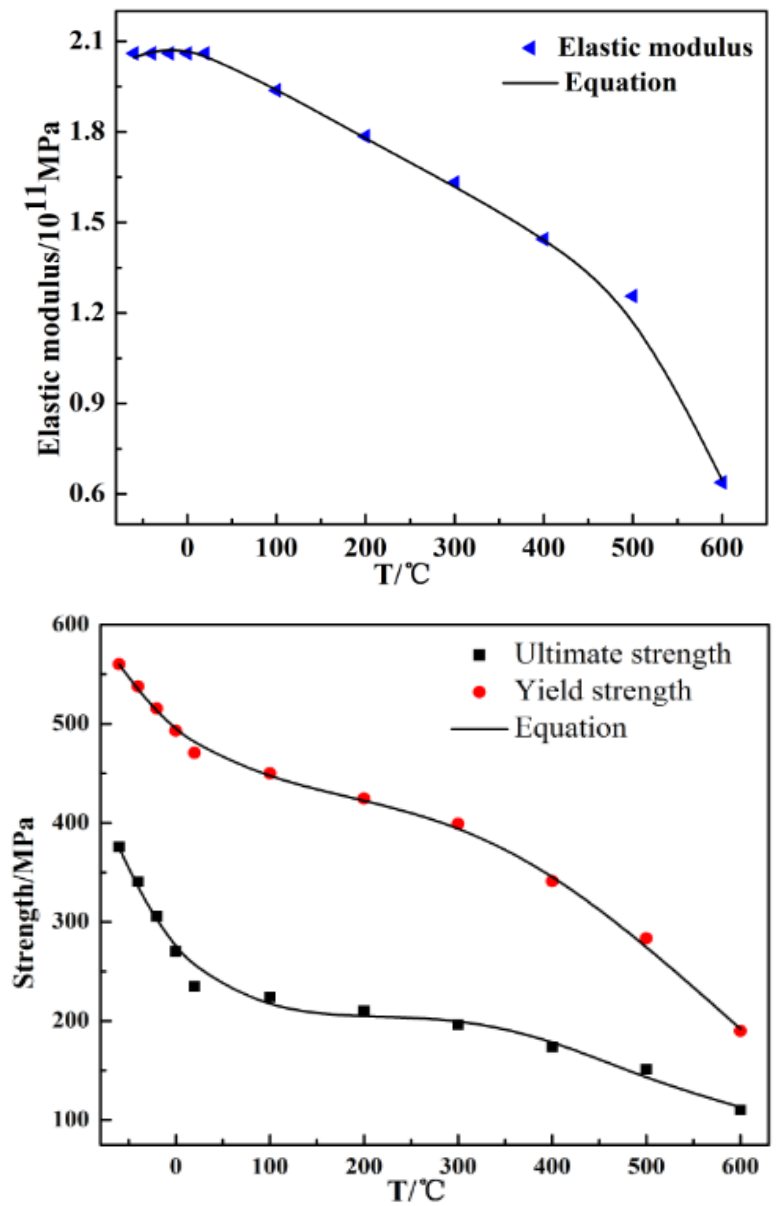

Figure 5 Comparison of fitting curves \& experimental values

BP neural network which is a multilayer feed forward neural network is based on error back propagation algorithm. The core of the BP algorithm is to correct the error of the back propagation, and then modify the weights of each neuron, so that the error signal is minimized, until the allowable error is satisfied to achieve the desired input and output mapping.

Based on the "neural network mapping model" of Q235 constitutive model, the above sample is trained and predicted, and the elastic modulus (yield strength, ultimate strength) at different temperatures are obtained as shown in table 3.

In order to compare the prediction effect of the support vector machine and neural network to the constitutive relation of Q235 steel, the results of two methods are compared. The results show that SVM prediction results are smaller than the neural network and the elastic modulus (yield strength, ultimate strength) has better fit, which demonstrates the superiority of support vector machine in the training of small sample data.

Table 3 BP mapping model predicted results

\begin{tabular}{rcccccc}
\hline Temperature $/{ }^{\circ} \mathrm{C}$ & -40 & -20 & 0 & 100 & 200 & 400 \\
\hline Elastic modulus $/ \mathrm{Pa}$ & $2.052 \mathrm{E}+11$ & $2.057 \mathrm{E}+11$ & $2.06 \mathrm{E}+11$ & $1.938 \mathrm{E}+11$ & $1.785 \mathrm{E}+11$ & $1.44 \mathrm{E}+11$ \\
Yield strength/MPa & 335.508 & 309.246 & 271.21 & 223.991 & 210.156 & 173.781 \\
Ultimate strength/MPa & 532.895 & 520.048 & 499.422 & 450.269 & 424.566 & 341.062 \\
\hline
\end{tabular}




\section{Conclusion}

(1)Through experimental study, the mechanical properties of Q235 steel were obtained. With the increase of temperature, the Ultimate strength, yield strength and elastic modulus of Q235 steel decreased. With the decrease of temperature, the Ultimate strength, yield strength and elastic modulus of Q235 steel showed a increasing trend, and the microstructure, hardness and strength of Q235 steel were almost no change at $-60^{\circ} \mathrm{C}$.

(2)Taking constitutive relation of Q235 steel as the object, stress-strain properties of Q235 steel were calculated and analyzed for different deformation temperatures, the constitutive relation which is convenient for engineering use are obtained by referring to British standard.

(3)By using SVM, the mapping relationship between input and output data is obtained by using the data of Q235 steel, and "mapping model" is established. The model is used to predict the elastic modulus (yield strength and ultimate strength) at different temperatures. Compared with the experimental results, the relative error is small, and model has a high degree of coincidence.

(4)Compared with neural network. The support vector machine (SVM) has a better fit for the prediction of elastic modulus (yield strength, ultimate strength), and proves the superiority of support vector machine in the training of small sample data.

\section{Reference}

[1] Xu Gening. Metal structure design of mechanical equipment[M]. 2nd Edition. Beijing: Mechanical industry press of China,2009.

[2] Dai Dongliang,Bu Xin. Study on stress-strain curve of steel at high temperature [J]. Journal of Luoyang Institute of technology,2011.21(1):14-18.

[3] Xu Yan,Zhao Jincheng. Experimental study on material properties of Q235 steel under different stress and temperature paths [J]. Journal of Shanghai Jiaotong University,2004,38(6):967-971.

[4] Li Guoqiang,Chen Kai,Jiang Shouchao, Yin Yingzhi. Experimental study on material properties of Q345 steel at high temperature [J]. Architectural structure,2001,31(1):53-55.

[5] Ou Manli, Cao Weijun. Study on mechanical properties of Q235 steel in high temperature (fire) [J]. Journal of Zhuzhou Institute of Technology, 2006，20(4):99-101.

[6] Cortes C,Vapnik V. Support vector network[J]. Machine Learning,1995,20: 273-297.

[7] Napnik V N.The Nature of Statistical Learning Theory [M]. New York: Springer,1995: 70-256.

[8] Hao P Y,Chiang J H.Fuzzy regression analysis by support vector learning approach[J]. IEEE Trans on Fuzzy Systems, 2008 16(2):428-441.

[9] Yang Zhimin,Liu Guangli. Principle and application of uncertainty support vector machine [M]. Beijing: Science press,2007.

[10] European committee for standardization NEN-EN 1993-1-2 eurocode 3-design of steel structures-part 1-2 General rules-structural fire design[S]

[11] Chen J, Young B. Stress-strain curves for stainless steel at elevated temperatures[J]. Engineering Structures, 2006,28 (2): 229-239 